Ann. Zootech., I963, 12 (I), 53-66

\title{
ESTIMATION DU RENDEMENT EN FROMAGE GRUYĖRE DE COMTÉ EN FONCTION DE LA RICHESSE DU LAIT DE CHAUDIÈRE
}

\author{
G. MOCQUOT, G. RICORDEAU, P. AURIOL \\ Station Centrale de Recherches laitières et de Technologie des produits animaux \\ et Station Centrale de Génétique animale, \\ Centre national de Recherches zootechniques, Jouy-en-Josas (Seine-et-Oise) \\ avec la collaboration technique de E. BLANC-l'atin et R. JEL'NeT, \\ Station expérimentale laitière, Poligny (Jwa) et de J. L. CANIYY
}

SOMMAIRE

C'ctte étude a pour but d'estimer le poids de fromage "Gruyère de Comté », qu'il est possible d'obtenir, en fonction de la richesse en matière grasse et cn protéines du lait de fabrication. Elle a été réalisée au cours d'une période de 6 années (1956-1962), dans une fromagerie du Jura.

Chaque mois, on a constitué un échantillon représentant l'ensemble des laits de chaudière et on a comptabilisé la production totale de fromage réellement fabriquée. Le poids de fromage obtenu à partir de roo $\mathrm{kg}$ de lait mis en cuvre (rendement) a été ensuite estimé à partir (tableau 3) : du taux butyreux (équation I) ; du taux de protéines (équation 2); du taux butyreux et du taux de protéines (équation 3).

L'écart type des différences entre le rendement cstimé (a l'aidle des 3 équations) et le rendement réel est respectivement de $220 \mathrm{~g}$ de fromage pour $100 \mathrm{~kg}$ de lait avec l'équation $\mathrm{I}$, de $165 \mathrm{~g}$ avec l'équation 2, et de $15 \mathrm{I} g$ avec la $3^{\mathrm{e}}$ équation. Dans cette dernière équation, le coefficicent de régression appliqué au taux de protéines est 3,8 fois plus élevé que celui appliqué au taux butyreux. La comparaison effectuée entre laits de producteurs et laits de " chaudière ", met en évidence l'intérêt d'une sélection en vue de l'accroissement du rapport protéines/graisse des laits de producteur, tout au moins dans les zones oi la fabrication de gruyère absorbe une part importante de la production laitière.

\section{INTRODUCTION}

Les relations entre le rendement en fromage d'un lait et sa composition sont intéressantes à connaître à double titre. Elles permettent, soit de prévoir la quantité de fromage qu'on peut obtenir à partir d'un lait de mélange de composition donnée, soit de déterminer l'importance relative des principaux constituants du lait pour la fabrication du fromage afin d'orienter le sélectionneur vers la production de laits présentant la composition jugée la plus favorable. 
Depuis 60 ans, différentes méthodes ont été proposées pour estimer le rendement en fromage.

La richesse en matière grasse du lait mis en fabrication a été le premier élément retenu : DEAx (I893), BEAU (I906), VAN SLyke (I9I7), ZEILER, BAUER et BERWIG (I928) VEALE (I929) WASSERMAN et JAGER (I953). Les premières équations obtenues se sont révélées insuffisantes par suite de la non-proportionnalité entre le rendement et le taux butyreux. Dans un deuxième stade, on a tenu compte, à la fois de la matière grasse et de l'extrait sec dégraissé du lait et du fromage. CASTELnAU (I92I), Vaillant (I933), Guerault (I956), VAn Dam et Janse (I931), Labianchy et I,ESCANnE (I947) ont alors proposé des formules plus complètes.

Un troisième stade a vu la prise en considération de la caséine (ou des matières azotées totales) du lait et de la richesse en matière grasse et en protéines du sérum. Ce stade, déjà clairement défini par VAN SLYKE (I894 b, Ig08), BABCock, FARRINGTON et HART (I9I0), a été précisé ensuite par VEALE (I933), UDy (I934), MCDOWALI, (I936), Sherton (I945), VAN Slyke et Price (I952), VALEN (I953), Jakubowski et BIJOK (I954), Schulz et KAY (I957). Les résultats de ces auteurs sont résumés dans le tableau I.

TABLEAU I

Équations donnant le rendement en fromage, en fonction des teneurs en protéines et matière grasse du lait de fabrication

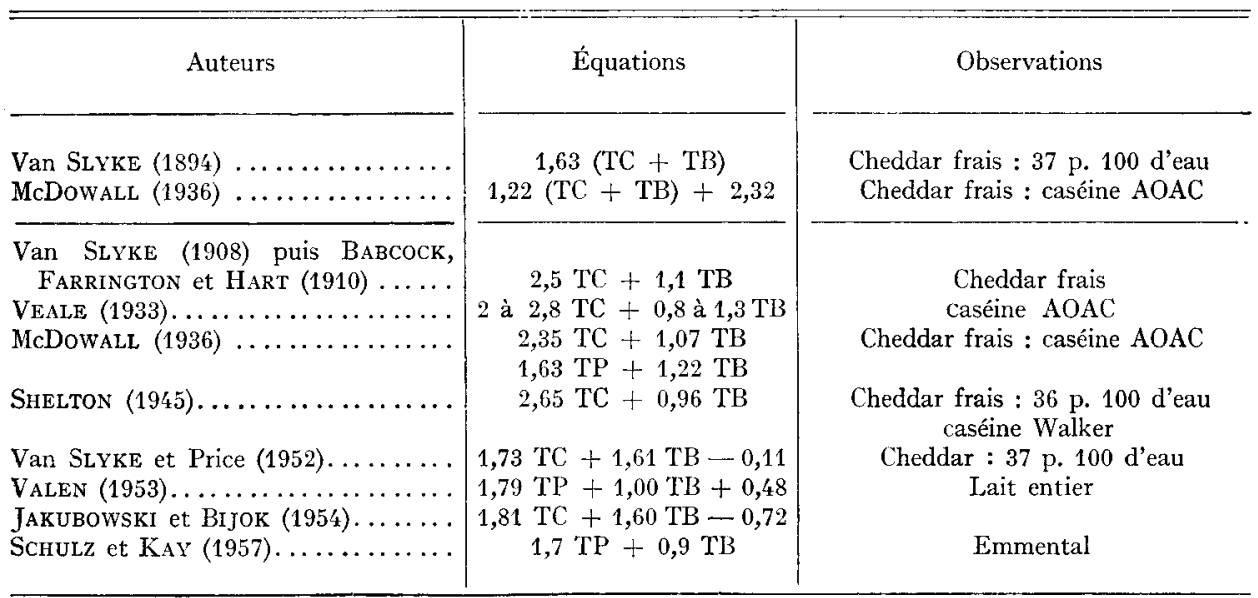

Les Cheddar sont fabriqués avec du lait entier. Pour les autres fromages, les laits utilisés étaient partiellement écrémés.

Les trois dernières formules (VALEN, Jakubowski et Bijok, Schulz et Kay), ont été obtenues en donnant des valeurs approximatives à certaines variables :

- matière grasse du sérum : $0,05 \mathrm{~g} \mathrm{p} .100 \mathrm{~g}$.

- extrait sec du fromage : $62,6 \mathrm{~g}$ p. $100 \mathrm{~g}$.

- fraction de la matière grasse du lait passant dans le fromage : $90 \%$

- fraction des protéines du lait passant dans le fromage : $75 \%$

Légende : $\quad \mathrm{TP}$ : taux de matières azotées totales

TC : taux de caséine

TB : taux de matière grasse 
Les équations de la forme " $k$ (matière grasse + caséine) " ou " $k$ (matière grasse + protéines )" se sont révélées les moins précises. Elles accordent en effet, la mëme importance aux deux éléments et ceci n'est pas exact si 1'on considère le rendement en fromage frais $\left({ }^{1}\right)$. Les autres équations sont déduites de la relation existant entre le rendement d'une part, les protéines et la graisse d'autre part, ces deux éléments étant affectés d'un coefficient tenant compte de leur importance relative; elles sont plus générales et plus précises que les précédentes. Dans toutes les équations appartenant à ce dernier type (et c'est cela qu'il est important de souligner), les coefficients de régression relatifs au taux butyreux sont inférieurs aux coefficients relatifs au taux de caséine ou de protéines. La caséine joue donc un rôle prépondérant dans le rendement en fromage, du fait qu'elle retient dans ce dernier beaucoup plus d'eau que les matières grasses (MCDowaL, I936; VAN SLYKE et PrICE, I952). Cette rétention d'eau par les protéines est d'ailleurs un phénomène très général. Il se traduit en particulier, par le fait que l'eau retenue par le fromage comprend à la fois de l'eau " libre " et de l'eau " liée "; les travaux de McDowal, et DolBy (I936), Pyenson et DAHLE (I938), Mocouot (I947), ont montré que l'eau "liée " était pour la plus grande part retenue par les protéines.

Comme nous pouvons le constater d'après le tableau I, les coefficients calculés pour la matière grasse et les protéines varient d'un auteur à l'autre. Ces écarts résultent de différences dans les techniques de fabrication et dans la richesse du lait utilisé ; chaque équation n'étant valable, en fait, que pour un type de fromage déterminé, fabriqué dans des conditions uniformes.

Partant de données observées dans le Jura, il nous a paru important de déterminer l'équation permettant de calculer le rendement en gruyère de Comté à partir de laits de mélange de richesse connue en graisse et protéines. Ce travail fait suite à l'étude entreprise sur la composition saisonnière des laits d'étable et de fromagerie du plateau de Nozeroy (AURIOL et MOCQUOT, I957). Il concerne des résultats obtenus dans la fromagerie de Censeau (Jura) de mars I956 à décembre I96r.

\section{MA'TÉRIEL F'T MÉTHODES}

\section{I. - FROMAGES}

La fabrication du Gruyère de Comté comprend plusieurs opérations. Le lait de chaudière (environ $55^{\circ} \mathrm{kg}$ par fromage), constitué par un mélange du lait de la traite du soir partiellement écrémé par gravité $\left(^{2}\right)$ et du lait de la traite du matin, est réchauffé à $33^{\circ} \mathrm{C}$ et additionné (à la dose de 0,3 p. Ioo) d'un levain lactique (composé de streptocoques et lactobacilles thermophiles) préparé selon le procédé traditionnel, dans une macération de caillettes de veaux qui apporte l'enzyme coagulant. 35 minutes après l'addition de présure, le lait coagulé est découpé (ro minutes), brassé et chauffé à $55^{\circ} \mathrm{C}$ (45 minutes), puis retiré du feu et encore brassé ( 55 minutes). Le caillé recueilli dans une toile (I 50 minutes après l'addition de présure) est placé dans un moule et pressé $\left(0, \mathrm{I} \mathrm{kg} / \mathrm{cm}^{2}\right.$ au début et $\mathrm{I} \mathrm{kg} / \mathrm{cm}^{2}$ à la fin) pendant 22 heures en opérant en moyenne 5 retournements. Chacun des chiffres cités représente une valeur moyenne et des fluctuations autour de cette valeur sont observées d'un jour à l'autre, d'une saison à l'autre, le travail du fromager étant toujours orienté vers l'obten-

(I) On entend ici par fromage c frais » (par opposition à l'extrait sec de fromage), le fromage qui contient la graisse, les protéines, les substances minérales et organiques variées et l'eau.

(2) Le lait de la traite du soir est laissé I 2 à ${ }_{15}$ heures au repos dans des bassines plates (" rondots »); on recueille alors la crème remontée en surface (" crème de ronde »). 
tion d'un fromage de composition (proportions respectives d'eau, de graisse et de protéines) aussi constante que possible.

A la sortie de la presse, 25 heures après l'emprésurage du lait, le fromage est transporté en cave, salé (avec du sel en gros grains déposé à la surface du fromage et aussi dans certains cas par immersion pendant 24 ou 48 heures dans un bain de saumure) et frotté pendant 4 à 6 mois à une température de $\mathrm{I} 6$ à $\mathrm{I} 8^{\circ} \mathrm{C}$. Le salage au gros sel et le frottage entraînent la formation d'un développement bactérien (morge) à la surface du fromage.

\section{2. - DONNÉES QUANTITATIVES RECUEILIIES A LA FROMAGERIE,}

Les enregistrements quotidiens portaient sur le poids total de lait livré par les producteurs et sur le poids des fromages à leur sortie de presse. Le poids mensuel de lait livré oscillait selon les mois de 40000 à II $5000 \mathrm{~kg}$ de lait; il est passé en moyenne de $60000 \mathrm{~kg}$ en 1956 à $80000 \mathrm{~kg}$ en I96I.

Les autres enregistrements concernaient le poids de lait vendu en nature et le poids de beurre obtenu, à partir de la " crème de ronde " et de la crème provenant de l'écrémage du lactosérum.

On a d'abord relevé le poids mensuel de lait vendu en nature qui représentait 2 ooo à $2400 \mathrm{~kg}$ ( 2 à $5 \mathrm{p}$. Ioo du poids total de lait livré, selon les mois).

On a ensuite évalué le poids de crème entrant mensuellement, dans la fabrication du beurre. Ce poids, a été calculé d'après les observations antérieures de l'un d'entre nous (MocQuot, 1943) selon lesquelles 65 p. Ioo du beurre obtenu provenait de la matière grasse de la "crème de ronde ", la crème contenant en moyenne $380 \mathrm{~g}$ de matière grasse par $\mathrm{kg}$, le beurre obtenu dosant $\mathrm{I4}$ p. Ioo d'eau. Le poids de beurre a donc été traduit en poids de crème, en utilisant le coefficient de transformation de I,4. En adoptant ce coefficient, le poids ainsi estimé représentait, au plus, 2 p. 100 du poids de lait livré ; les erreurs susceptibles d'être introduites dans l'utilisation du coefficient étaient donc négligeables.

Le poids total de lait livré, diminué du poids de lait vendu en nature et du poids de crème (estimé comme il vient d'être dit), représentait le poids de lait de chaudière transformé en fromage.

\section{3. - PrélìtVements des ǴChantilions \\ ET ANALYSES EFFECTUÉES AU LABORATOIRE}

\section{Lait de chaudiere}

A chaque fabrication, on prélevait un échantillon du lait de chaudière. Le mélange de tous ces échantillons unitaires a permis de constituer, pour chaque mois, un échantillon représentatif de l'ensemble des laits de chaudière. Les analyses des échantillons mensuels concernaient les éléments suivants : la matière grasse, dosée selon le procédé Gerber; les matières azotées, dosées selon la méthode Kjeldahl (teneur en $\mathrm{N} \times 6,38$ ).

\section{Laits des producteurs}

La composition du lait a été suivie dans cinq étables de la même commune (Censeau). Les échantillons, prélevés lors des deux livraisons quotidiennes de chaque producteur et représentant le lait de chaque traite pour chacune des étables contrôlées, étaient additionnés de formol (o, I p. Ioo de formol à $40 \mathrm{p}$. Ioo). Tous les mois, on prélevait, sur chaque échantillon journalier, une quantité de lait proportionnelle à la quantité livrée par le producteur. Le mélange de tous les prélèvements proportionnels permettait d'obtenir pour chaque étable, un échantillon mensuel, sur lequel on effectuait, comme ci-dessus, les déterminations de matières azotées et de matières grasses.

\section{RÉSULTATS}

Le graphique I traduit l'ensemble des variations mensuelles et annuelles de la richesse des laits (de producteurs et de chaudière) et du rendement en fromage. Le tableau 3 donne les caractéristiques de chaque variable et les différentes équations obtenues. 


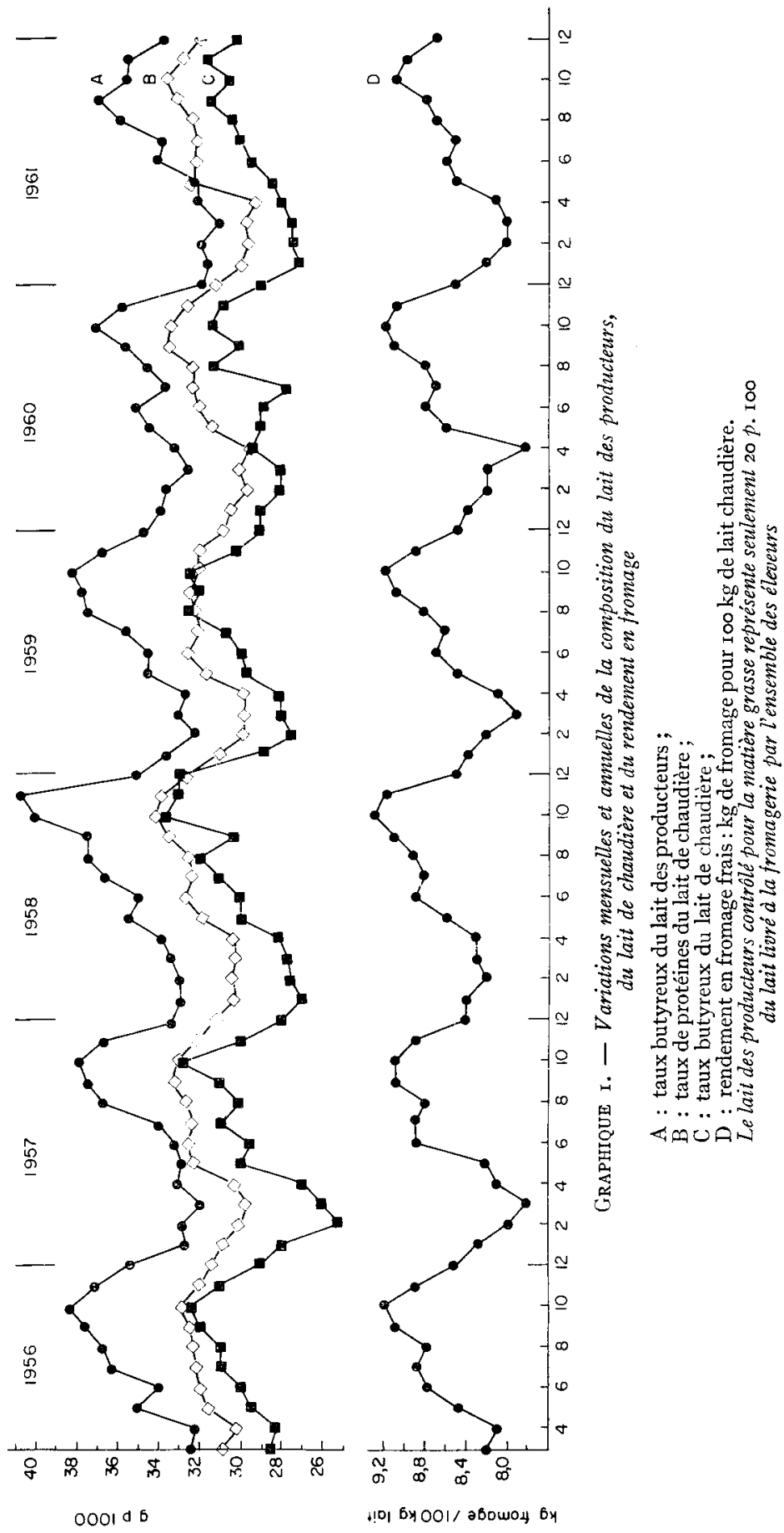


Le rendement d'un lait en fromage ( $z=$ poids de fromage obtenu à partir de Ioo $\mathrm{kg}$ de lait) est fonction de la richesse de ce lait en protéines ( $x$ ) et en matière grasse $(y)$. Nous pouvons étudier séparément la relation entre $z$ et $x$, $z$ et $y$, mais nous verrons plus loin qu'il existe une relation positive entre $x$ et $y$.

Pour essayer de séparer les influences respectives de ces deux facteurs ( $x$ et $y$ ) dans la variation de $z$, nous pouvons étudier la régression de $z$ sur chaque couple ( $x$ et $y$ ). Cette méthode de la régression multiple a l'avantage de donner une solution pour chaque valeur des deux variables perturbatrices, quel que soit le rapport entre ces deux variables. En fait, l'utilisation des valeurs du triplet $(z, x, y)$ suppose la vérification d'un certain nombre d'hypothèses de base : linéarité des régressions de $z$ sur $x, z$ sur $y$ et de $z$ sur $x$ et $y$; unicité des variances de $z$ liées par $x, y$ ou $x$ et $y$.

Lorsque les hypothèses précédentes ne sont pas parfaitement vérifiées, il semble préférable de calculer le rendement en fromage, non plus pour roo $\mathrm{kg}$ de lait $(z)$ mais pour I $\mathrm{kg}$ de matière grasse utilisée $(Z)$. Il suffit ensuite d'étudier les variations de $Z$ en fonction du rapport protéines/matière grasse. Cette solution, préconisée par MCDowal, (I936), a l'avantage de permettre le calcul direct de $z$ en fonction de $x$ et $y$ (le rendement $z$ étant cependant indéterminé pour le cas, d'ailleurs peu fréquent à l'époque actuelle, de fromages fabriqués avec des laits très pauvres en matière grasse ou même complètement écrémés).

$Z$, poids de fromage par $\mathrm{kg}$ de matière grasse $=k_{1} x / y+k_{2} ;$ puisque $z$, poids de fromage pour Ioo $\mathrm{kg}$ de lait $=Z y$ on a : $z=k_{1} x+k_{2} y$.

En tenant compte du rapport $\frac{x}{y}$ au lieu du couple $(x, y)$ on suppose implicitement que le rendement $Z$ est le même pour des laits de différentes richesses en graisse ou protéines mais de même rapport $x / y$. Cette hypothèse est valable avec les laits de chaudière du Jura. Les analyses effectuées par l'un d'entre nous (Mocquo'T, r963) du lactosérum de 2 I échantillons de "laits riches » et de 27 échantillons de "laits pauvres » de même rapport $x / y$ indiquent en effet des pertes sensiblement égales (en valeur relative) pour les deux catégories de lait (tableau 2).

TABLEAU 2

Richesse de deux catégories de laits de chaudière et des lactosérums correspondants (en $\mathrm{g} p .1000 \mathrm{~g}$ )

\begin{tabular}{|c|c|c|c|c|c|c|c|c|}
\hline \multirow{2}{*}{$\begin{array}{l}\text { Valeurs moyennes } \\
\text { correspondant } \\
\text { à }\end{array}$} & \multicolumn{3}{|c|}{ Lait } & \multicolumn{3}{|c|}{$\begin{array}{l}\text { Lactosérum de fin } \\
\text { de brassage }\end{array}$} & \multicolumn{2}{|c|}{$\begin{array}{l}\% \text { de protéines et de } \\
\text { graisse du lait passées } \\
\text { dans le lactosérum* }\end{array}$} \\
\hline & $\mathrm{TP}$ & TB & $\mathrm{TP} / \mathrm{TB}$ & $\mathrm{TP}$ & TB & $\mathrm{TP} / \mathrm{TB}$ & Protéines & Graisse \\
\hline 21 " laits riches" & 33,1 & 32,7 & 1,01 & 8,7 & 5,9 & 1,47 & 24,5 & 16,8 \\
\hline 27 " laits pauvres $" \ldots \ldots \ldots$ & 30,0 & 29,7 & 1,01 & 7,9 & 5,4 & 1,46 & 26,0 & 17,9 \\
\hline
\end{tabular}

* Ce pourcentage a été calculé, déduction faite du poids de matière sèche du fromage. 
TABLEAU 3

Rendement en Gruyère frais et richesse en protéines et matière grasse du lait de chaudière

\begin{tabular}{|c|c|c|c|c|c|}
\hline \multicolumn{2}{|c|}{ Equations } & \multirow{2}{*}{$\begin{array}{c}r \\
r_{z y}=0,82\end{array}$} & \multirow{2}{*}{$\frac{r^{2}}{(p \cdot 100)}$} & \multirow{2}{*}{$\begin{array}{c}\text { Droite de régression estimée } \\
z^{\prime}=3,372+0,176 y\end{array}$} & \multirow{2}{*}{$\begin{array}{c}\begin{array}{c}\text { Écart-type } \\
\text { résiduel* } \\
s_{z-z_{i}}\end{array} \\
0,220\end{array}$} \\
\hline 1 & $z_{1}^{\prime}=a_{1}+b_{z y} \cdot y$ & & & & \\
\hline 2 & $z_{2}^{\prime}=a_{2}+b_{z x} \cdot x$ & $r_{z x}=0,90$ & 81 & $z^{\prime}=-0,354+0,283 x$ & 0,165 \\
\hline 3 & $z_{3}^{\prime}=a_{3}+b_{z x, y} \cdot x+c_{z y}, x \cdot y$ & $r_{z}, x_{y}=0,92$ & 85 & $\begin{aligned} z^{\prime}=0,079 & +0,216 x \\
& +0,056 y\end{aligned}$ & 0,151 \\
\hline 4 & $z_{4}^{\prime}=k_{1} x+k_{2} y$ & & & $z^{\prime}=0,218 x+0,058 y$ & 11.150 \\
\hline
\end{tabular}

70 observations réparties sur 6 ans. Chaque échantillon correspond à 1 mois de fabrication.

* Estimation sans biais de l'écart-type résiduel en $\mathrm{kg}$ de fromage pour $100 \mathrm{~kg}$ de lait.

\begin{tabular}{|c|c|c|c|c|}
\hline & Variable & Moyenne & Écart-type & Limites de variation \\
\hline Rendement (kg de fromage pour $100 \mathrm{~kg}$ de lait) $: \ldots$ & $\ldots z$ & 8,61 & 0,39 & 7,7 a $9, \dot{x}$ \\
\hline Richesse du lait , protéines (g p. $1000 \mathrm{~g}$ )..... & $\ldots x$ & 31,68 & 1,26 & 29,6 a 34,2 \\
\hline de chaudière matière grasse (g p. $1000 \mathrm{~g}$ ) & $\ldots y$ & 29,70 & 1,84 & 25,2 a 33,8 \\
\hline
\end{tabular}

\section{Prévision du rendement (z) à partir de la richesse en matière grasse du lait de chaudière $(y)$. Graphique $2 \mathrm{~A}$}

La ligne de régression estimée de $z$ en fonction de $y$ (équation I) est linéaire pour $y$ variant de 25 à $34 \mathrm{~g}$ p. I ooog. L'écart-type de $z$ lié par $y\left(s_{z}^{\prime}\right)$ est de $0,220 \mathrm{~kg}$ de fromage pour $100 \mathrm{~kg}$ de lait.

2. Prévision du rendement (z) à partir de la richesse en matière azotée du lait de chaudière $(x)$. Graphique $2 \mathrm{~B}$

La ligne de régression de $z$ sur $x$ est parfaitement linéaire pour $x$ variant de 20 à $35 \mathrm{~g}$ p. I ooo g (équation 2). A partir de cette régression, l'estimation de l'écarttype résiduel $(s z)$ n'est plus que de $0,165 \mathrm{~kg}$.

\section{Prévision du rendement (z) à partir de la richesse en protéines $(x)$ et de la richesse en matière grasse $(y)$ du lait de chaudière.}

Les deux équations précédentes ne permettent pas de préciser l'influence respective des deux facteurs $x$ et $y$ dans la détermination de $z$. En tenant compte à la fois des deux variables, il est possible d'augmenter la précision de $z$.

A partir du modèle linéaire à deux variables $\left(z^{\prime}=a+b x+c y\right)$ nous avons calculé la droite de régression estimée (équation 3 ).

L'estimation sans biais de l'écart-type résiduel $\left(s_{z}^{\prime}\right)$ est alors de $0, I_{5} \mathrm{I} \mathrm{kg}$ de 


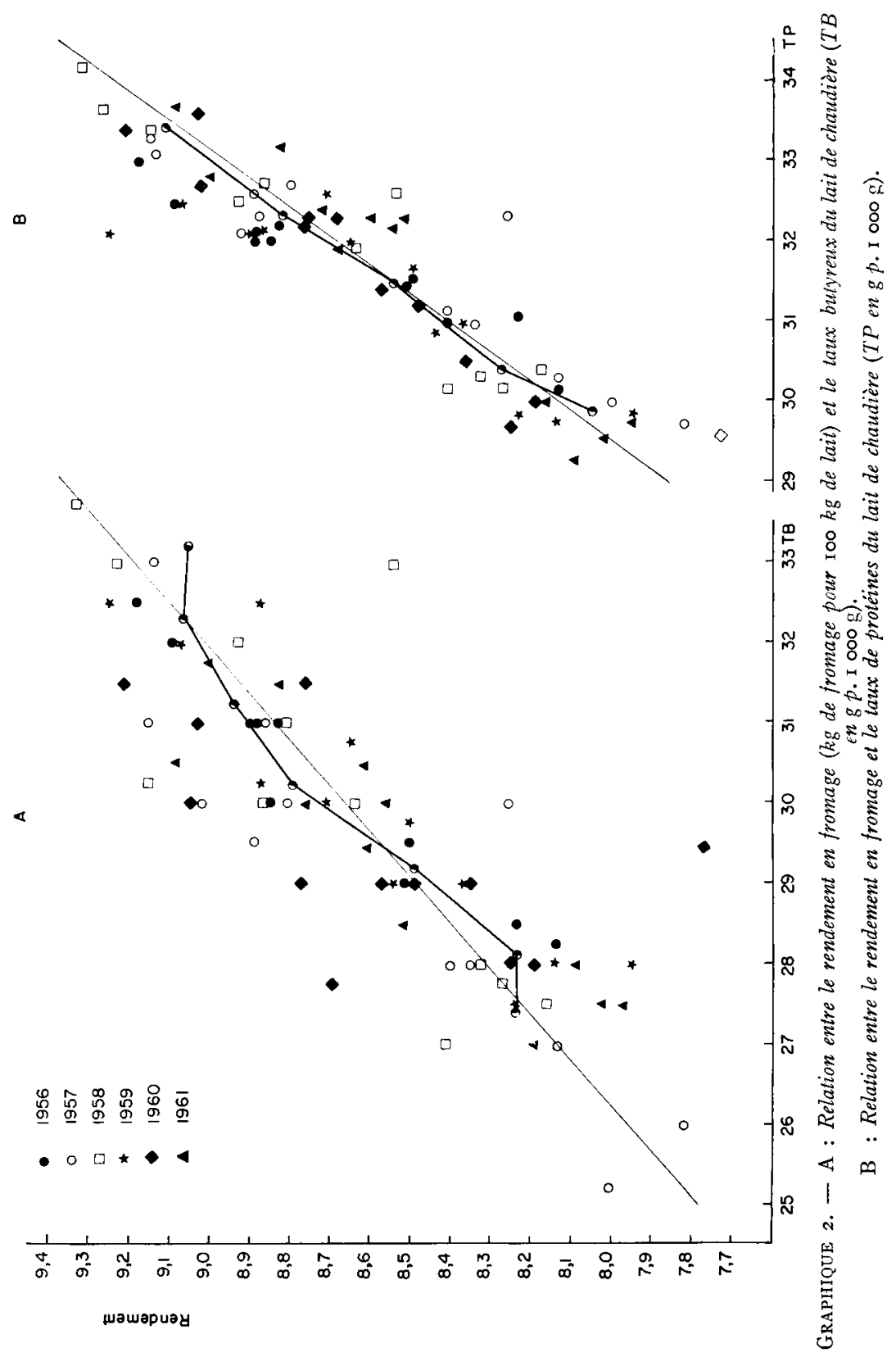


fromage par Ioo $\mathrm{kg}$ de lait. Les écarts $\left(z_{i}^{\prime}-z^{\prime}\right)$ ayant une distribution normale et une variance indépendante de $x$ et $y$, on peut donc prévoir $z$ à partir de $x$ et de $y$ avec une erreur au seuil de $95 \mathrm{p}$. Ioo de l'ordre de $\pm 0,296 \mathrm{~kg}$ de fromage $\left( \pm \mathrm{t}_{67 ; 2,5 \%} \cdot \mathrm{s}_{z}^{\prime}\right)$.

La précision des coefficients de régression $b$ et $c$ est assez faible $\left(s_{b}=0,0245\right.$, $s_{c}^{\prime}=0,0160 j$, ce qui n'est pas étonnant, puisque les estimations de $b$ et $c$ sont d'autant plus imprécises (les dispersions marginales de $x$ et $y$ étant constantes) que le coefficient de corrélation $r_{x y}$ est plus élevé.

\section{Production de fromage par $\mathrm{kg}$ de matière grasse et rapport protéines/graisse du} lait de chandière.

Le poids de fromage par $\mathrm{kg}$ de matière grasse mise en œuvre $(Z)$ est en relation liné-

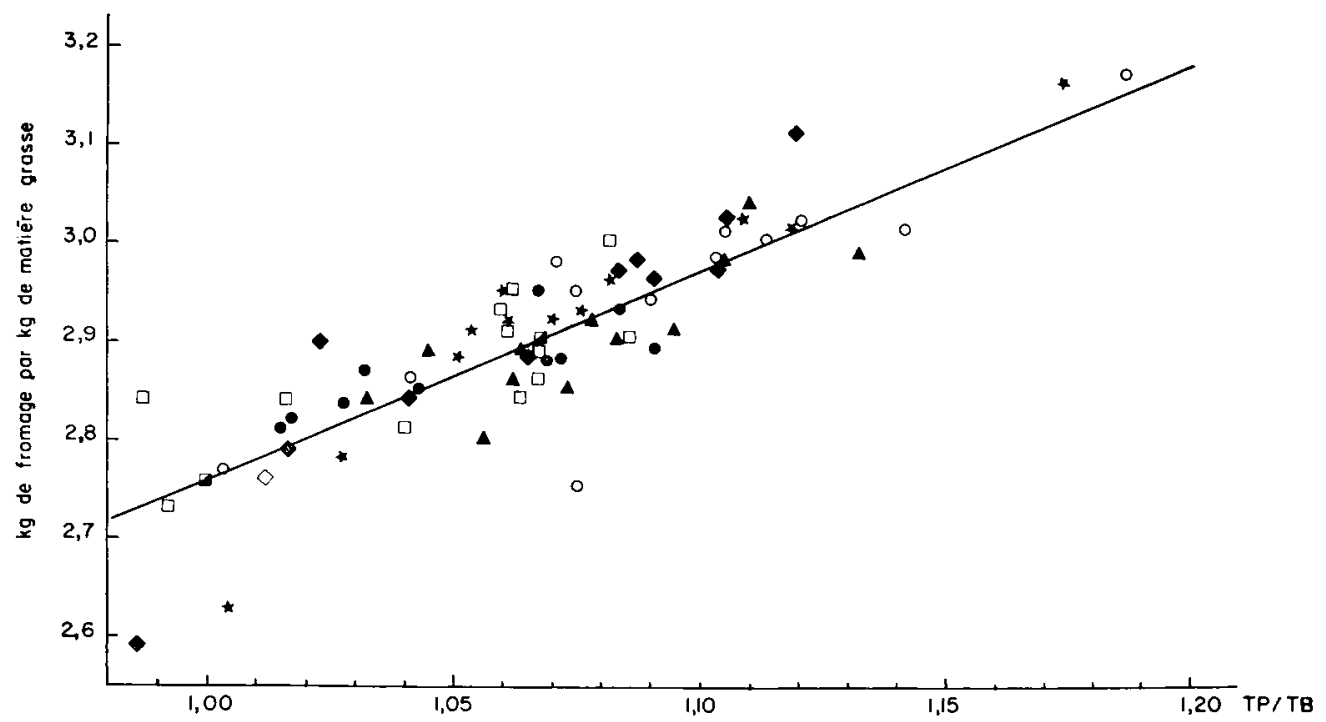

GRAPHiQUe 3. - Production de fromage par kg de matière grasse en fonction du rapport protéines/graisse du lait de chaudière

aire avec le rapport $\frac{x}{y}(r=0,86)$. La droite donnant l'estimée de $Z$ est :

$$
Z^{\prime}=0,058+0,2 \mathbf{I} 8 x / y
$$

d'oì $z_{4}^{\prime}=0,2 \mathrm{I} 8 x+0,05^{8} y$ avec $x$ et $y$ eng p. I ooo g (équation 4 ).

Équations I,2 et 3 .

\section{Comparaison des quatre équations}

En définissant l'efficacité comme le rapport des variances liées, on peut calculer que l'estimation $\left(z_{3}\right)$ de $z$ à partir de $x$ et de $y$ est respectivement 2,I3 et I, I5 fois plus efficace que les estimations $\left(z_{1}\right)$ et $\left(z_{2}\right)$.

L'estimation $\left(z_{2}\right)$ est elle-même $\mathrm{I}, 85$ fois plus efficace que l'estimation $\left(z_{1}\right)$ En se basant uniquement sur le taux de protéines, on obtient donc une très bonne estimation du rendement. On peut se demander si l'amélioration de précision obtenue lorsqu'on tient compte en outre de la richesse en matière grasse du lait est significative. 
Le test du coefficient $c$ relatif à $y\left(c / s_{c}^{\prime}\right)$ donne une valeur de 3,36 hautement significative au seuil I p. Ioo. Le rendement (z) dépend donc bien de $x$ et de $y$. C'est l'étroite relation entre ces deux variables dans les conditions pratiques de fabrication à la fromagerie de Censeau $\left(r_{x y}=0,80\right)$ qui ne permet pas de séparer parfaitement leur influence respective. Puisque $x$ et $y$ jouent dans le même sens, en se basant uniquement sur $y$, on attribue à cette variable une partie de 1 'influence due à $x$ et inversement. C'est ce qui explique que les coefficients de régression de $z$ sur $y\left(b_{z y}\right)$ ou $z$ sur $x\left(b_{z x}\right)$ soient respectivement supérieurs aux coefficients $c$ et $b$ de la régression multiple à 2 variables.

Equations 3 et 4 .

Les deux équations donnent le même résultat. La méthode de la régression multiple fait intervenir une constante, mais cette dernière est pratiquement négligeable.

A Censeau, d'après l'ensemble des résultats mensuels obtenus sur six années, on peut donc prédire le rendement en $\mathrm{kg}$ de fromage frais $(z)$ avec une erreur de \pm $0,300 \mathrm{~kg}$ de fromage pour Ioo $\mathrm{kg}$ de lait, dans $95 \mathrm{p}$. Ioo des cas, selon l'une ou l'autre des deux formules suivantes :

$$
\begin{aligned}
& z=0,080+0,2 \mathrm{I} 6 x+0,056 y \\
& z=0,218 x+0,058 y
\end{aligned}
$$

dans lesquelles, rappelons-le, taux de protéines $(x)$ et taux butyreux $(y)$ sont exprimés en $g$ de (protéines ou de graisse) pour I ooo $g$ de lait.

\section{Observations concernant la relation entre $\mathrm{x}$ et $\mathrm{y}$}

Les teneurs en protéines des laits de chaudière sont égales à celles des laits de producteurs, mais les teneurs en matière grasse diffèrent, car les laits de chandière résultent, nous l'avons dit, d'un écrémage partiel du lait livré. En prenant comme base la richesse moyenne du lait de 5 éleveurs fournissant environ $20 \mathrm{p}$. Ioo de la quantité totale de lait utilisée à la fromagerie, cet écrémage abaisse le taux butyreux d'au moins $6 \mathrm{~g}$ de graisse pour I ooo g de lait. Alors que le rapport $x / y$ du lait livré est en moyenne de 0,88 , il est de $\mathrm{I}, 07$ pour le lait de chaudière. Pour presque tous les mois considérés, ce lait de chaudière était plus riche en protéines qu'en matière grasse. A Censeau, en dépit des variations mensuelles importantes de la richesse du lait, le fromager a toujours écrémé de façon que le rapport protéines/graisse de son lait de chaudière varie relativement peu (le coefficient de variabilité de ce rapport est de 3,7 p IOO.).

La régression de $x$ sur $y$ est linéaire $\left(b_{x y}=0,55\right.$ pour l'ensemble des 70 observations mensuelles), mais les distributions marginales de $x$ et $y$ sont plutôt d'allure bimodales, par suite des fluctuations saisonnières très marquées, notamment pour le taux de protéines. Les régressions de $z$ sur $x$ ou de $z$ sur $x$ lié par $y$ sont cependant linéaires, comme nous avons pu le vérifier sur de petits échantillons ayant même teneur en matière grasse.

\section{Variations de l'écart entre rendement observé et rendement théorique en fonction $d u$ mois de fabrication}

L'analyse de la variance des écarts entre rendements observés et rendements théoriques calculés selon l'équation 4 , ne révèle pas de différence significative sui- 
vant le mois de l'année $\left(F=I, 7 ; v_{1}=I I, v_{2}=68\right)$ bien que, par rapport aux rendements théoriques attendus, on constate une perte en mars-avril-mai et un gain en septembre-octobre-novembre.

Bien que non significatives, les différences observées au cours de ces deux périodes correspondent peut-être aux variations saisonnières de la teneur en caséine du lait (Mocolot, I956, BERGMaN et Joost, I953) et par suite, du pourcentage de protéines entraînées dans le caillé (Jensen, Pedersen, OvergaArd, I954).

\section{DISCUSSION}

I e rendement en fromage est conditionné, essentiellement, par la compétence professionnelle du fromager et par des facteurs relevant de la composition du lait en fabrication.

A Censeau, pendant six années, le même fromager a travaillé de façon uniforme. La qualité de son travail et l'importance de chaque "échantillon mensuel " (40 à I I 2 tonnes de lait) permettent de supposer que les variations aléatoires ont été réduites au minimum. La composition du lait (richesse en protéines et matière grasse) permet done d'expliquer une grande partie de la variance totale du rendement : $85 \mathrm{p}$. Ioo en utilisant l'équation 3 , mais aussi $8 \mathrm{I}$ p. roo à l'aide de l'équation 2 qui tient uniquement compte de la richesse en protéines du lait de chaudière, richesse qui reflète celle des laits d'éleveurs. I)ans les conditions bien précises d'obtention des laits de chaudière destinés à la fabrication du Comté, la teneur en protéines des laits livrés permet donc de calculer une bonne estimation du rendement en fromage frais, c'està-dire du poids de fromage que produit et vend directement le producteur.

Dans l'équation 3 (tableau 3) qui donne la meilleure estimation du rendement, le coefficient de régression $b$ relatif au taux de protéines est 3,8 fois plus élevé que le coefficient de régression $c$ relatif au taux butyreux. Ce résultat confirme les travaux antérieurs cités au début de cet exposé, en particulier ceux précisant le rôle des protéines comme agents de fixation de l'eau (eau liée et eau libre) du fromage. Le rapport de 3,8 est cependant supérieur à ceux que l'on peut déduire des équations du tableau I qui concernent presque toutes le fromage de Cheddar. Bien qu'il soit dangereux d'extrapoler les résultats de chacune de ces équations en dehors des domaines de variation couverts par les variables ayant servi à leur calcul, on peut, semble-t-il, expliquer les écarts observés, par des différences dans la composition des fromages résultant de la composition du lait et par des différences dans les techniques de fabrication. En effet, le découpage en grains assez fins du caillé de gruyère et la température atteinte au cours de la cuisson $\left(52\right.$ à $\left.5^{6} \mathrm{C}\right)$ sont autant de facteurs favorisant les pertes en matière grasse dans le sérum, alors que, pour une même variation du rapport TP/TB, le découpage du caillé en grains plus gros et les températures de cuisson et de pressage plus basses du Cheddar, entraînent une perte moindre de graisse dans le lactosérum. Dans le cas de fabrication d'Emmental, Schulz et KAY (I957) aboutissent à une équation qui ne doit pas être significativement différente de la nôtre, si l'on se souvient que les vraies valeurs de $b$ et $c$ ne sont pas connues, mais sont comprises avec une probabilité de 95 p. Ioo dans les intervalles suivants : 
0,167 à 0,256 pour $b$ et 0,022 à 0,090 pour $c$. On peut également penser qu'avec des laits de grand mélange, la faible variabilité des teneurs en graisse et surtout en protéines limite la signification des coefficients $b$ et $c$. En fait, nous avons pu vérifier, sur un échantillon récent de 60 laits de chaudière (correspondants chacun à un seul fromage) dont les teneurs en protéines et en graisse variaient toutes deux de 27 à $3^{6} \mathrm{~g} \mathrm{p}$. I ooog que le rendement était estimé par une équation très voisine.

La question des taux respectifs de protéines et de graisse du lait se rattache à un autre problème : celui de savoir si, dans une région fromagère comme le Jura par exemple, il convient de fabriquer à partir d'un poids déterminé de lait, un poids maximum d'un fromage réputé (gruyère de Comté) et une quantité relativement faible de beurre (de qualité souvent médiocre et de commercialisation difficile) ou s'il faut conserver la proportion actuelle de beurre et de fromage. La solution de ce problème dépend à la fois de facteurs économiques et techniques.

Sur le plan économique, une étude globale s'impose pour déterminer notamment les prix respectifs du gramme de protéines et du gramme de graisse.

Sur le plan technique, on doit tenir compte des considérations suivantes :

Io) Pour obtenir un bon gruyère de Comté, il faut un lait de chaudière dont la teneur en protéines soit à peu près égale à la teneur en graisse : un lait trop pauvre en graisse donne un fromage qui " manque de pâte » (on sait d'ailleurs que le minimum imposé est de $45 \mathrm{p}$. Ioo de matière grasse dans la substance sèche du fromage) et un lait trop riche en graisse augmente les risques de lainure.

$\left.2^{\circ}\right)$ On a vu dans cette étude que le lait livré possède une richesse en graisse nettement supérieure à sa richesse en protéines : c'est donc la richesse en protéines, et non la richesse en matière grasse, qui constitue le facteur limitant la production de fromage (tout le poids de graisse en excès du poids de protéines devant être transformé en beurre).

Si finalement, on estime souhaitable de fabriquer relativement plus de fromage et moins de beurre, il faut augmenter annuellement la quantité de protéines produite par les vaches du Jura, sans augmenter - ou en augmentant dans une proportion plus faible - la quantité de graisse. Un tel but doit pouvoir être atteint, en suivant un programme de sélection axé sur un accroissement de la quantité de lait, de la teneur en protéines ainsi que du rapport protéines/graisse du lait. Il semble bien en effet, comme le constatent Von Krosigk, Young et Richardson (I960), Pirchner, Stuber et TSCHORNER (I962), que (par suite de la variabilité différente des teneurs en graisse et protéines) la sélection sur le seul taux de protéines se traduise à long terme, par une amélioration corrélative du taux butyreux légèrement supérieure à celle obtenue pour l'élément directement sélectionné, ce qui reviendrait encore à diminuer le rapport protéines/graisse. Au contraire, la sélection sur ce rapport aurait peu de chances de provoquer une diminution de la quantité de lait (1'accroissement du rapport protéines/graisse correspond à une diminution du coût énergétique de la production) et permettrait en outre d'exercer une certaine sélection négative sur la richesse en matière grasse. Il s'agit cependant de maintenir un taux butyreux satisfaisant au niveau des laits de mélange, notamment par élimination des vaches donnant des laits trop pauvres en graisse au cours des premiers mois de lactation.

En s'imposant une limite minimum pour le taux butyreux et en sélectionnant ensuite les anima11x sur le taux de protéines et le rapport protéines/graisse, deux paramètres dont 1'héritabilité est supérieure à 0,4 (HANsson, I956; Aurior, I962), 
on devrait aboutir à une modification de la proportion entre matière grasse et protéines en faveur de ces dernières. Ces conclusions provisoires demandent cependant à être confirmées par des études génétiques portant sur un plus vaste matériel et en tenant compte du niveau de production laitière. A notre connaissance en effet, si la corrélation génétique entre quantité et lait et taux butyreux est bien connue et se révèle presque toujours négative, celle existant entre quantité de lait et taux de protéines n'a fait l'objet que de rares investigations (ROBERTSON, WAITE et WHITE, I956).

$$
\text { Ręu pour publication en février } 1963 .
$$

\section{REMERCIEMENTS}

Nous remercions le président, le fromager et les producteurs de la fruitière de Censeau de l'aide qu'ils nous ont apportée pour ce travail.

\section{SUMMARY}

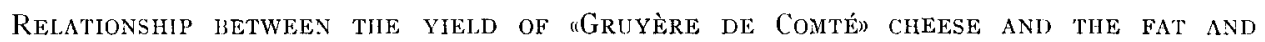
PROTEIN CONTENT OF TIE «KETTLE» MILK.

A study of the relationship between the weight of " Gruyère de Comté " cheese and the fat and protein content of the milk from which it was manufactured was carried out during the period i9561962 at a cheese factory, in the Jura district.

Every month a sample of milk, constituted by representative samples from all the "kettles " in the factory, was collected and analysed and the total weight of cheese manufactured and the weight of milk received at the factory were recorded. The cheese weight obtained from $100 \mathrm{~kg}$ of milk (cheese yield) was then estimated (table 3) from the butterfat content (first equation), from the protein content (second equation) and from both the butterfat and protein contents (third equation).

The standard deviation of the differences between the yield for roo $\mathrm{kg}$ of milk estimated (from the three equations) and the actual yield is respectively $220 \mathrm{~g}$ of cheese for the first equation, ${ }^{6} 65 \mathrm{~g}$ for the second equation and $\mathrm{I} 5 \mathrm{I} g$ for the third equation. In the third equation, the regression coefficient applied for the protein content is 3.8 times higher than the one applied for butterfat content. The comparison between producers' milk and "kettle" milk. makes it obvious that the selection should aim at increasing the protein/fat ratio in producer's milk, at least in those districts where Gruyère manufacture utilises a large part of the milk produced.

\section{RÉFÉRENCES BIBLIOGRAPHIQUES}

Auriol P., Mocquot G., 1957. Variations saisonnières de la composition des laits d’étable et de fromagerie dans la région du gruyere. I. Plateau de Nozeroy (Jura). Ann. Zootech., 2, 95-1 20.

Auriol P., 1962. Comment améliorer, à la production, la teneur du lait en protéines. Bull. tech. Ing. Serv. agric., 168, 3-20.

BEAU M., rgo6. Sur le payement du lait d'après sa richesse. Lail, 6, 93-94.

Babcock S. M., Farrington E. H., HaRt E B., rgio. Methods of paying for milk at cheese factories. Univ. Wis. Agric. Exp. Bull., 197, 3-24.

BERGMAN T., JoOST K., I953. Variations in the protein content of the cheese milk and its composition during differe it periods of the year, as well as the effect of the said variations on the cheese yield and on the composition of the cheese. $X I I I^{\circ}$ Congr. int. Laiterie, La Haye, 2, 83-88. 
CASTELAaU R., I921. Le payement du lait suivant sa richesse totale. Lait, 10, 526-528.

VAN DAM W., JANSE L. C., I93I. The relation between fat and casein contents of milk of separate farms and the significance thereof in the matter of payment at butter and cheese factories. Versl. Rijkslandb, Proefst's Grav., 31 (cité par McDowall, 1936).

Dean H. H., 1894. Experiments in Checsemaking. Ont. Agr. Coll. Bull., 95 (cité par McDowall, 1936).

MCDowall F. H., I936. The cheese yielding capacity of milk and its relation to the method of payment for milk for cheesemaking. Dairy Res. Inst. N.Z., 72, I $37 \cdot 354$.

McDowall F. H., Dolby R. M., 1936. Studies on the chemistry of cheddar cheese making. J. Dairy Res., $7, \mathrm{I}_{5} 6-\mathrm{I} 75$.

Guerault R., 1956. La fromagerie devant les techniques nouvelles. Édition S. E. P. Paris.

Hansson A., 1956. Genetic variation in the content of milk constituents. VII e Congr. int. Zootech. Madrid., 5, I I I-I I 7 .

JakUbowski J., Bijok, 1954. Détermination du rendement en fromage en fonction de la composition chimique du lait (en polonais). Z. Zakladu, Technologii, kierononik mgr. Jan. Cesul, I9-3o.

Jensen Hi., Pedersex A. H., Overgaard S., i954. Bereln Forsoksm kbh., 86, 9-204.

Krosigk (von) C. M., Young J. O., Richardson G. A., I g6o. Genetic influences on the composition of cow's milk. J. Dairy Sci., 43, 877 (Abstr.).

Lablanchy A., Lescanne R., I947. Études sur les rendements en fromagerie. Sci., Tech., Econ. lait., 1, I I $9^{-1} 37$.

MocQuot G., I947. Étude sur l'eau liée des fromages. Lait, 27, 576-595.

Mocquot G., 1956. Cité dans JARrige R. Variations de la teneur en matières azotées des laits individuels. VII Congr. Int. Zootech. Madrid, I39.

MocQuot G., 1963. Résultats non publiés.

Pirchner F., Stuber O., Tschorner F., ig62. Vererbung von Milchbestandteilen bei Höhenvieh. $Z$. Tierzücht. Ziicht. Biol., 77, 285*296.

Pyenson H., Dahle C. D., 1938. Bound water and its relation to some dairy products. J. Dairy. Sci., 21, $169-185$.

Robertson A., Watte R., White J. C. D., 1956. Variations in the chemical composition of milk with particular reference to the solids not fat. Il. The effect of heredity. J. Dairy Res., 23, 82-9I.

Schulz M. E., KAY H., I957. Käse-Tabellen : Eiweisstiter, Fetteinstellung, Käsesollausbente. Milchw. Verlag Th. Mann K. G. Hildesheim.

Shelton A. B., I945. Cheddar cheese composition and yield in relation to fat and casein content of milk. Publication of N.S.W Dept. of Agriculture. Division of Dairying issue.

Van Slyke L. L., I884 b. N. Y. Agr. Expt. Sta. Bull, 65 (cité dans VAn Slyke et Price, I952).

VAN SlyKe L. L., I908. Conditions affecting the proportions of fat and proteins in cow's milk. J.Amer. Chem. Soc., 30, I I66-I I86.

Van Styke L. L., igr7. Methods of paying for milk at cheese factories. N. Y. Agr. Exp. Sta. Bull., 308 (cité par McDowalL, 1936).

Van Slyke L. L., Price W. V., 1952. Cheese., Orange Judd. Pub. Co, New York.

Udy W. H. (Cité par McDowall, I936).

VAILLANT M. E., 1933. Une méthode simple, pratique et rationnelle de payement du lait fromager. Lait, 13, $487-495$.

VALEN A. L., I953. The relation between fat and protein in milk delivered to cheese factories in Norway and the significance of this relationship for cheese making. XIII ${ }^{\circ}$ Congr. int. Laiterie, La Haye, 3, I $47 \mathrm{I}^{-}$ I 477 .

Veale P. O., I929 et I933. (Cité par McDowall, I936).

Wasservann O., Jager H., 1953. Fettgehalt der Kesselmilch und Käseausbeute. XIII Congr. int. Laiterie, La Haye, 2, 732.736.

Zeiler K., Bauer H., Berwig A., I928. Estimation et payement du lait selon sa qualité (cité dans Lait, 89, 970-973).

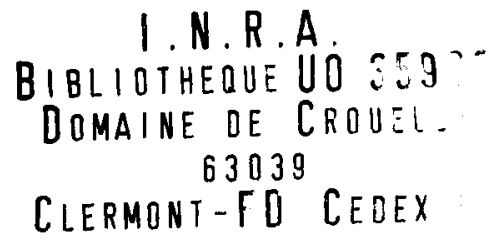

